\title{
Musculcutanous Sarcoidosis Following Intramuscular Injection of Synthol; Case Study
}

\author{
Medhat Ahmed Fouad Shalaby*, Mamdooh Ahmed Khairy, Sameh Hassan al Zayat, \\ Samy Abd Alhakim
}

Rheumatology Department, Faculty of Medicine, AL-Azhar University, Cairo, Egypt

Email: *medshalaby@hotmail.com

How to cite this paper: Shalaby, M.A.F., Khairy, M.A., al Zayat, S.H. and Alhakim, S.A. (2022) Musculcutanous Sarcoidosis Following Intramuscular Injection of Synthol; Case Study. Journal of Biosciences and Medicines, 10, 51-57.

https://doi.org/10.4236/jbm.2022.102006

Received: December 26, 2021

Accepted: February 8, 2022

Published: February 11, 2022

Copyright $\odot 2022$ by author(s) and Scientific Research Publishing Inc. This work is licensed under the Creative Commons Attribution International License (CC BY 4.0).

http://creativecommons.org/licenses/by/4.0/

\begin{abstract}
Sarcoidosis is a non caseating granulomatous disease of unknown etiology. Many factors may be attributed to the occurrence of that granuloma including the chemical factors. We present this case aiming to highlight Synthol injection which is chemical substance frequently used by athletics to increase their muscle size as it can trigger the occurrence of musculocutanous sarcoidosis. In conclusion Synthol should be added to the chemical list which can induce musculocutanous sarcoidosis.
\end{abstract}

\section{Keywords}

Sarcoidosis, Synthol

\section{Introduction}

Sarcoidosis is a multisystem disorder of unknown etiology characterized by the accumulation of $\mathrm{T}$ lymphocytes, mononuclear phagocytes, and noncaseating granuloma in involved tissues [1] [2]. The disease usually begins in the lungs, which are affected in approximately 90 percent of patients. And the upper lobes of the lung and bronchovascular bundles are usually affected more than other lung compartments. Lung involvement is often associated with hilar and mediastinal lymphadenopathy [3]. Other organs like skin and lymph nodes are commonly affected by sarcoidosis. Less commonly organs are affected including the eyes, liver, heart, and brain. However any organ, can be affected. And the signs and symptoms depend on the organ involved [4]. The cause of sarcoidosis is still unknown. Some believe it may be due to an immune reaction to a trigger such as an infection or chemicals in those who are genetically predisposed [5]. A well 
known chemical substance called Synthol is a used by body builders as a temporary implant which is injected deeply into the muscle to enlarge its volume. The muscles which are frequently injected include triceps, biceps, deltoids and muscles of the calf. The enlargement effects are usually immediate [6]. Synthol consists usually of oil, benzyl alcohol and lidocain. It consists of $85 \%$ of oil (normally it is oil built by medium-length MTC chains because it gives the best effects), $7,5 \%$ of lidocain (painkiller), 7, 5\% of alcohol (to sterilize the mixture) [6]. Some serious drawbacks can be visible while using Synthol. The muscles deform and become unnaturally shaped. The side effects of Synthol are manifold and they can also cause a damage of nerves, oil embolic of the pulmonary, occlusion of the pulmonary artery, myocardial infarction, cerebral stroke and infectious complications [6]. We present this case that developed musculocutanous sarcoidosis after Synthol injection

\section{Case Presentation}

In December 2016, A 40 year old Egyptian male presented to the ER with high grade fever $\left(39^{\circ}\right)$, and dry irritating cough. He admitted for work up. He gave past history of receiving intramuscular injection of Synthol in both triceps muscle during his gymnastic activities to increase the bulk of his muscle 6 months ago. Clinical examination and laboratory tests at the time of admission were unremarkable except elevated ESR ( $80 \mathrm{mmhg}$ ). And plain X-ray chest was normal. The patient received cough depressor and antipyretics, improved and the fever subside within one week and discharged as a case of bronchitis. February 2017, the patient was travelling to UAE (United Arab of Emirate) where he admitted to the Rheumatology unit as he got another attack of high grade fever with pain and swelling in his right elbow. On examination (from their report) he was febrile (temp 39 degree), vital signs were normal including blood pressure. Neck examination revealed scattered lymph node enlargement. Musculosketal examination showed swollen tender right elbow with erythematous skin lesion extended from the shoulder to the right elbow and enlarged multinodular soft tissue swelling in the bake of right arm. No other joint was clinically affected. Laboratory investigations include ESR (60 mmhg) CRP $8.5 \mathrm{mg} / \mathrm{dl}$, Hepatitis screening, liver function tests and renal function tests were normal, HIV screening was also negative. Serum vitamin D was 33.85 (normal), total and ionized serum calcium were normal $(9.7,5.1 \mathrm{mg} / \mathrm{dl})$ respectively. Immune panel s for ANA, RF, Anti Ds-DNA, Scl-70, and Anti-centromere were normal. Abdominal ultrasonography and CT chest were done and also were normal. He was diagnosed as localized Scleroderma (Morphea) based on clinical and laboratory examination (but without skin biopsy) and started immunosuppressive therapy with Methotrexate $2.5 \mathrm{mg}$ tablet (4 tablet/week), and corticosteroid $10 \mathrm{mg} /$ day. The patient showed improvement as regards to the fever and right elbow effusion but the triceps became more bulky with multiple non tender nodular enlargements and his skin was still erythematous. At the end of February, 2017 the patient got similar attack in the left elbow (swollen, tender with effusion) with erythematous 
skin lesion in the back of left arm extended up to the shoulder and down to the elbow, with development of multiple non tender nodular swelling in the back of left arm mainly triceps muscle (Figure 1(a) \& (Figure 1(b)).

So, the skin lesion was biopsied and the result showed non caseating granulomatous reaction extending from mid-dermis to the lower margin of the biopsy specimen. The granuloma was rounded to oval, composed of collections of epitheloid cells and multinucleated giant cells (MNGCs). They are surrounded with minimal lymphocytes. They extend around sweat gland structures and some foamy appearance of the cells was perceived in one level examined. Modified ZN stain for lepra bacilli was negative. The final conclusion that the histopathological features together with histochemical features are consistent with sarcoidosis (Figure 2).

CT scan with contrast for neck, chest, abdomen and pelvis showed hyper metabolic cutanous, subcutaneous and inter-muscular thickening of the shoulders, arms and back of the neck as well as bilateral axillary lymph node enlargement (Figure 3). F-FDG PET/CT examination showed clear both lung fields with no pulmonary nodules, acinar or interstitial abnormalities (Figure 4) and increased 18-F-FDG uptake in cutanous, subcutaneous and inter-muscular area with soft tissue thickening involving the shoulders, arms and back of the lower neck with SUV max 11.3 o the left side. Also few bilateral axillary lymph node showing increased 18F-FDG uptake with SUVmax 5.1 on the right side. No hypermetabolic mediastinal lymphadenopathy or other hypermetabolic lesion throughout the body (Figure 4 \& Figure 5).

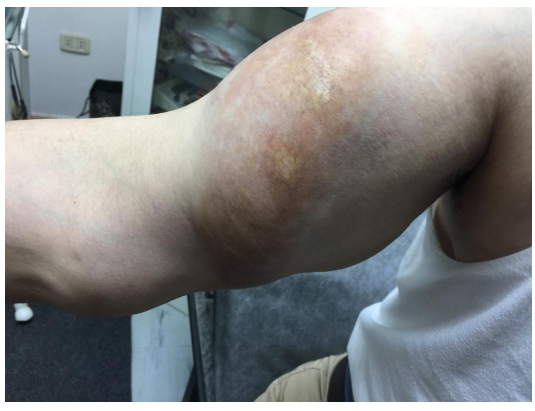

(a)

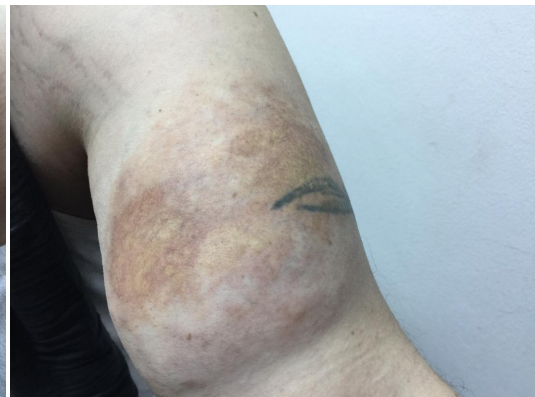

(b)

Figure 1. Swollen, tender left elbow (a) with erythematous skin lesion in the back of left arm extended up to the shoulder and down to the elbow, with development of multiple non tender nodular swelling in the back of left arm mainly triceps muscle $(a, b)$.
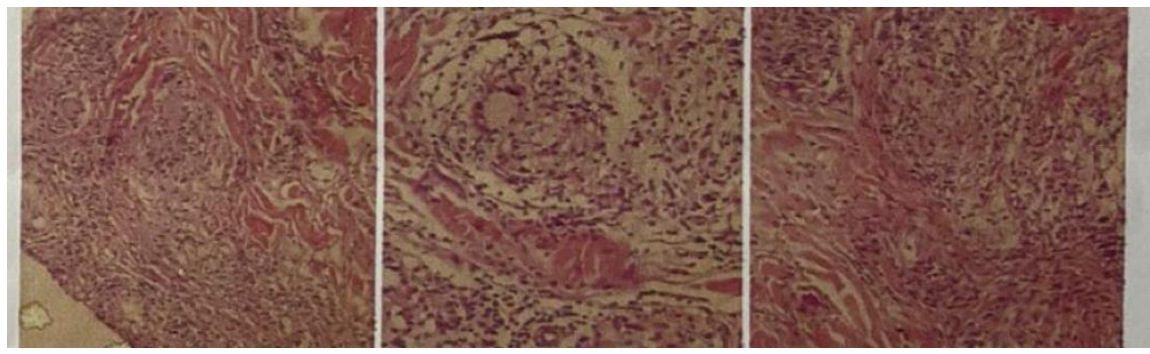

Figure 2. Skin biopsy showed non caseating granulomatous reaction extending from mid-dermis to the lower margin of the biopsy specimen. 

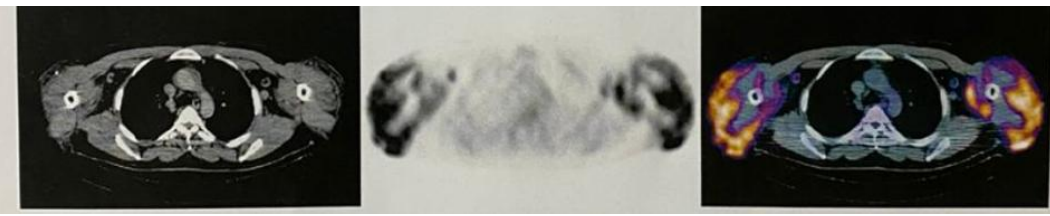

Figure 3. CT scan with contrast for neck, chest, abdomen and pelvis showed hyper metabolic cutanous, subcutaneous and inter-muscular thickening of the shoulders, arms and back of the neck as well as bilateral axillary lymph node enlargement.
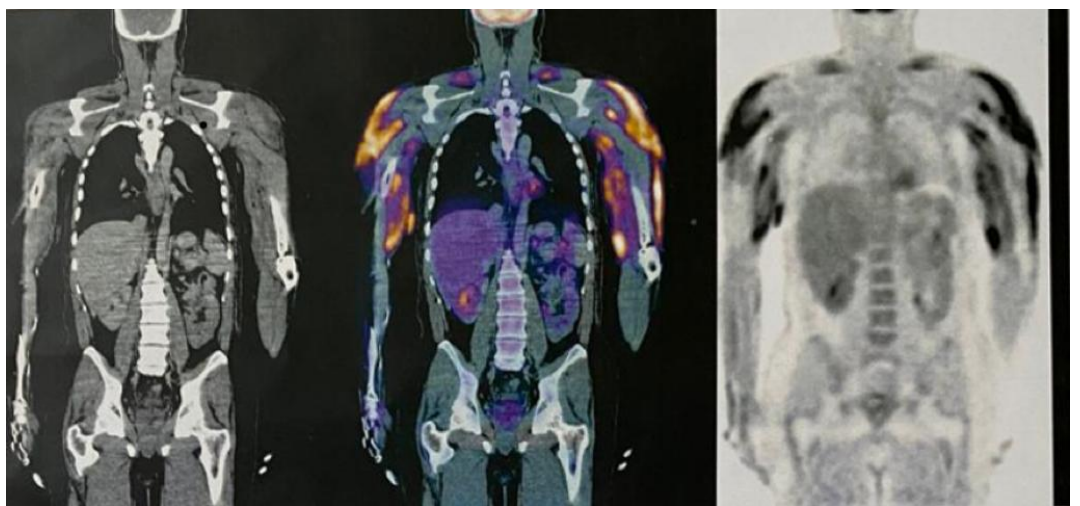

Figure 4. PET scan showed clear both lung fields with no pulmonary nodules, acinar or interstitial abnormalities.

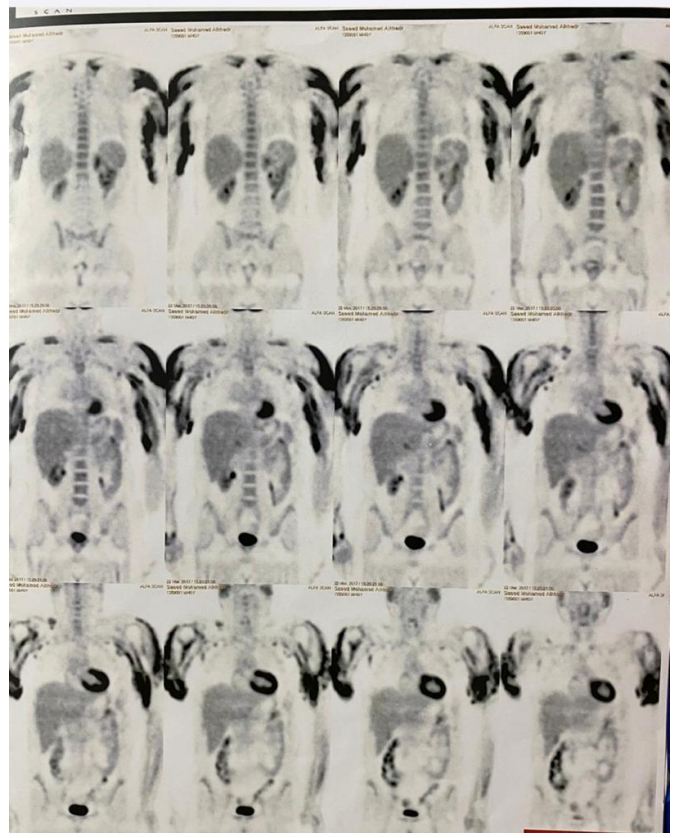

Figure 5. Showed increased 18-F-FDG uptake in cutanous, subcutaneous and inter-muscular area with soft tissue thickening involving the shoulders, arms and back of the lower neck few bilateral axillary lymph node.

The final diagnosis was made as musculocutanous sarcoidosis. The following treatment was started, Azathioprin $50 \mathrm{mg}$ BID, prednisolone $5 \mathrm{mg}$ BID, colchicines 0.5 mg BID. Last review in December 2021, his condition was stable, his 
laboratory tests showed mild elevation of ACE (60 u/l), CRP (1.65 mg/L), normal serum level for creatinine, LFT, CBC and ESR. He didn't get any new attack of joint pain nor swelling, non febrile, no another new lesion within the triceps muscle.

The patient was informed and consent was taken $\mathrm{f}$ or publication his case.

\section{Discussion}

Sarcoidosis (also known as Besnier-Boeck-Schaumann disease) is a chronic inflammatory multisystem disease characterized by granulomatous formation. Any organ, can be affected but the disease usually begins in the lungs, skin, or lymph nodes, less commonly, the eyes, liver, heart, and brain. And although it is more than 140 years had been passed since Hutchin-son (I875) [7] described his first case of coetaneous sarcoidosis, its etiology has not been satisfactorily explained and is still a matter of lively debate [2]. Some believe it may be due to an immune reaction to a trigger such as an infection or chemicals in those who are genetically predisposed [4] [8]. The proposed mechanism for sarcoidosis is that a susceptible genotype is exposed to one or more potential antigens. A sustained inflammatory response follows, which ultimately results in pathognomonic granuloma formation. Various clinical phenotypes exist with specific genetic associations influencing disease susceptibility, protection, and clinical progression. Occupational and environmental factors, including microbial elements, may then affect the development of this disease [9]. Many risk factors may be associated with sarcoidosis. Obesity, The Black Women's Health Study, a follow-up study of 59,000 African-American women, found that obesity [body mass index (BMI) $\left.30 \mathrm{~kg} / \mathrm{m}^{2}\right]$ at study baseline was associated with a $40 \%$ increased incidence of sarcoidosis over a 16-year period (1995-2011; HR 1.42; 95\% CI 1.07 - 1.89) [10]. Also crystalline silica is an occupational exposure released in mining, construction, and agricultural work. Silica has been shown to be associated with several autoimmune diseases such as systemic sclerosis, rheumatoid arthritis, systemic lupus erythematosus, and Antineutrophil cytoplasmic antibody (ANCA)-related vasculitis [11]. A recent cohort study conducted in silica-exposed workers in Sweden found that high exposure to silica dust $\left(>0.048 \mathrm{mg} / \mathrm{m}^{3}\right)$ was associated with an increased risk of sarcoidosis compared with those with less exposure (standardized incidence ratio 3.94; 95\% CI 1.07 - 10.08) [12]. As regards to smoking, one of the strongest associations to emerge from the ACCESS study was a 35\% reduced odds of sarcoidosis among ever smokers compared with never smokers [13]. A few studies had been published about the complication of Synthol intramuscular injection, Suleiman Ghandourah et al. (2012) reported a case of bodybuilder patient how got Painful muscle fibrosis following Synthol injections [14]. Another case reported by Andrea Sisti et al. (2020), patient with severe side effects following repeated intramuscular injections of Synthol in his right biceps muscle. The patient presented with extensive inflammatory changes throughout the anterior compartment of the musculature and lateral aspect of the deltoid 
musculature bilaterally, compatible with myositis, fasciitis, and cellulites [15]. To my knowledge, my case is the first case that presented with musculoskeletal sarcoidosis, another complication which should be added to the side effects of Synthol intramuscular injection.

\section{Conflicts of Interest}

The authors declare no conflicts of interest regarding the publication of this paper.

\section{References}

[1] Baughman, R.P., Lower, E.E. and du Bois, R.M. (2003) Sarcoidosis. Lancet, 361, 1111-1118. https://doi.org/10.1016/S0140-6736(03)12888-7

[2] Thomas, P.D. and Hunninghake, G.W. (1987) Current Concepts of the Pathogenesis of Sarcoidosis. The American Review of Respiratory Disease, 135, 747.

[3] NHLBI (2013) What Are the Signs and Symptoms of Sarcoidosis?

[4] Baughman, R.P., Culver, D.A. and Judson, M.A. (2011) A Concise Review of Pulmonary Sarcoidosis. American Journal of Respiratory and Critical Care Medicine, 183, 573-581. https://doi.org/10.1164/rccm.201006-0865CI

[5] NHLBI (2013) What Causes Sarcoidosis?

[6] Pupka, A., Sikora, J., Mauricz, J., Cios, D. and Płonek, T. (2009) The Usage of Synthol in the Body Building. Polymers in Medicine, 39, 63-65.

[7] Hutchinson, J. (1898) Archives of Surgery. Hutchinson, 9, 307.

[8] Siltzbach, L.E. (1958) The Etiology of Sarcoidosis. Postgraduate Medical Journal, 34, 254-256+258+267. https://doi.org/10.1136/pgmj.34.391.254

[9] Dubrey, S., Shah, S., Hardman, T. and Sharma, R. (2014) Sarcoidosis: The Links between Epidemiology and Etiology. Post Graduated Medical Journal, 90, 1086. https://doi.org/10.1136/postgradmedj-2014-132584

[10] Cozier, Y.C., Coogan, P.F., Govender, P., et al. (2015) Obesity and Weight Gain in Relation to Incidence of Sarcoidosis in US Black Women: Data from the Black Women's Health Study. Chest, 147, 1086-1093. https://doi.org/10.1378/chest.14-1099

[11] Miller, F.W., Alfredsson, L., Costenbader, K.H., et al. (2012) Epidemiology of Environmental Exposures and Human Autoimmune Diseases: Findings from a National Institute of Environmental Health Sciences Expert Panel Workshop. Journal of Autoimmunity, 39, 259-271. https://doi.org/10.1016/j.jaut.2012.05.002

[12] Vihlborg, P., Bryngelsson, I.L., Andersson, L., et al. (2017) Risk of Sarcoidosis and Seropositive Rheumatoid Arthritis from Occupational Silica Exposure in Swedish Iron Foundries: A Retrospective Cohort Study. BMJ Open, 7, e016839. https://doi.org/10.1136/bmjopen-2017-016839

[13] Newman, L.S., Rose, C.S., Bresnitz, E.A., Rossman, M.D., Barnard, J., Frederick, M., Terrin, M.L., Weinberger, S.E., Moller, D.R., McLennan, G., Hunninghake, G., DePalo, L., Baughman, R.P., Iannuzzi, M.C., Judson, M.A., Knatterud, G.L., Thompson, B.W., Teirstein, A.S., Yeager, H., Jr Johns, C.J., Rabin, D.L., Rybicki, B.A. and Cherniack, R. (2004) ACCESS Research Group. A Case Control Etiologic Study of Sarcoidosis: Environmental and Occupational Risk Factors. American Journal of Respiratory and Critical Care Medicine, 170, 1324-1330.

[14] Ghandourah, S., et al. (2012) Painful Muscle Fibrosis Following Synthol Injections 
in a Bodybuilder: A Case Report. Journal of Medical Case Reports, 6, Article No. 248. https://doi.org/10.1186/1752-1947-6-248

[15] Sisti, A., Huayllani, M.T., Restrepo, D.J., Boczar, D., Manrique, O.J., Broer, P.N., Shapiro, S.A. and Forte, A.J. (2020) Oil Injection for Cosmetic Enhancement of the Upper Extremities: A Case Report and Review of Literature. Acta Biomedica Atenei Parmensis, 91, e2020082. 